\title{
Two-dimensional enrichment analysis for mining high-level imaging genetic associations
}

\author{
Xiaohui Yao · Jingwen Yan - Sungeun Kim - Kwangsik Nho • \\ Shannon L. Risacher • Mark Inlow • Jason H. Moore • \\ Andrew J. Saykin $\cdot$ Li Shen
}

Received: 21 January 2016/Accepted: 29 April 2016/Published online: 13 May 2016

(C) The Author(s) 2016. This article is published with open access at Springerlink.com

\begin{abstract}
Enrichment analysis has been widely applied in the genome-wide association studies, where gene sets corresponding to biological pathways are examined for significant associations with a phenotype to help increase statistical power and improve biological interpretation. In this work, we expand the scope of enrichment analysis into brain imaging genetics, an emerging field that studies how genetic variation influences brain structure and function measured by neuroimaging quantitative traits (QT). Given the high dimensionality of both imaging and genetic data, we propose to study Imaging Genetic Enrichment Analysis (IGEA), a new enrichment analysis paradigm that jointly considers meaningful gene sets (GS) and brain circuits (BC) and examines whether any given GS-BC pair is enriched in a list of gene-QT findings. Using gene
\end{abstract}

For the Alzheimer's Disease Neuroimaging Initiative: Data used in preparation of this article were obtained from the Alzheimer's Disease Neuroimaging Initiative (ADNI) database (adni.loni.usc.edu). As such, the investigators within the adni contributed to the design and implementation of ADNI and/or provided data but did not participate in analysis or writing of this report. A complete listing of ADNI investigators can be found at: http://adni.loni.usc.edu/wpcontent/uploads/how_to_apply/adni_acknowledgement_list.

X. Yao · J. Yan · S. Kim · K. Nho · S. L. Risacher · M. Inlow · A. J. Saykin · L. Shen $(\bowtie)$

Radiology and Imaging Sciences, Indiana University School of Medicine, 355 West 16th Street Suite 4100, Indianapolis, IN, USA e-mail: shenli@iu.edu

X. Yao

School of Informatics and Computing, Indiana University Indianapolis, Indianapolis, IN, USA

J. H. Moore

Biomedical Informatics, School of Medicine, University of Pennsylvania, Philadelphia, PA, USA expression data from Allen Human Brain Atlas and imaging genetics data from Alzheimer's Disease Neuroimaging Initiative as test beds, we present an IGEA framework and conduct a proof-of-concept study. This empirical study identifies 25 significant high-level two-dimensional imaging genetics modules. Many of these modules are relevant to a variety of neurobiological pathways or neurodegenerative diseases, showing the promise of the proposal framework for providing insight into the mechanism of complex diseases.

Keywords Imaging genetics · Enrichment analysis · Genome-wide association study - Quantitative trait

\section{Introduction}

Brain imaging genetics is an emerging field that studies how genetic variation influences brain structure and function. Genome-wide association studies (GWAS) have been performed to identify genetic markers such as single nucleotide polymorphisms (SNPs) that are associated with brain imaging quantitative traits (QTs) [20, 21]. Using biological pathways and networks as prior knowledge, enrichment analysis has also been performed to discover pathways or network modules enriched by GWAS findings to enhance statistical power and help biological interpretation [6]. For example, numerous studies on complex diseases have demonstrated that genes functioning in the same pathway can influence imaging QTs collectively even when constituent SNPs do not show significant association individually [18]. Enrichment analysis can also help identify relevant pathways and improve mechanistic understanding of underlying neurobiology $[7,11,15,19]$. 
In the genetic domain, enrichment analysis has been widely studied in gene expression data analysis and has recently been modified to analyze GWAS data. GWASbased enrichment analysis first maps SNP-level scores to gene-based scores, and then tests whether a pre-defined gene set $S$ (e.g., a pathway) is enriched in a set of significant genes $L$ (e.g., GWAS findings). Two strategies are often used to compute enrichment significance: thresholdbased [4, 5, 9, 24] and rank-based [23]. Threshold-based approaches aim to solve an independence test problem (e.g., chi-square test, hypergeometric test, or binomial $z$ test) by treating genes as significant if their scores exceed a threshold. Rank-based methods take into account the score of each gene to determine if the members of $S$ are randomly distributed throughout $L$.

In brain imaging genetics, the above enrichment analysis methods are applicable only to genetic findings associated with each single imaging QT. Our ultimate goal is to discover high-level associations between meaningful gene sets (GS) and brain circuits (BC), which typically include multiple genes and multiple QTs. To achieve this goal, we propose to study Imaging Genetic Enrichment Analysis (IGEA), a new enrichment analysis paradigm that jointly considers sets of interest (i.e., GS and BC) in both genetic and imaging domains and examines whether any given GS-BC pair is enriched in a list of gene-QT findings.

Using whole brain whole genome gene expression data from Allen Human Brain Atlas (AHBA) and imaging genetics data from Alzheimer's Disease Neuroimaging Initiative (ADNI) as test beds, we present a novel IGEA framework and conduct a proof-of-concept study to explore high-level imaging genetic associations based on brain wide genome-wide association study (BWGWAS) results. For consistency purpose, in this paper, we use GS to indicate a set of genes and $\mathrm{BC}$ to indicate a set of regions of interest (ROIs) in the brain. The proposed framework consists of the following steps (see also Fig. 1): (1) conduct BWGWAS on ADNI amyloid imaging genetics data to identify SNP-QT and gene-QT associations, (2) use AHBA to identify meaningful GS-BC modules, (3) perform IGEA to identify GS-BC modules significantly enriched by geneQT associations using a threshold-based strategy, and (4) visualize and interpret the identified GS-BC modules.

\section{Methods and materials}

We write matrices and vectors as bold uppercase and lowercase letters, respectively. Given a matrix $\mathbf{M}=\left[m_{i j}\right]$, we denote its $i$ th row as $\mathbf{m}^{i}$ and $j$ th column as $\mathbf{m}_{j}$. Given two column vectors $\mathbf{a}$ and $\mathbf{b}$, we use $\operatorname{corr}(\mathbf{a}, \mathbf{b})$ to denote their Pearson's correlation coefficient.
2.1 Brain Wide Genome-Wide Association Study (BWGWAS)

The imaging and genotyping data used for BWGWAS were obtained from the Alzheimer's Disease Neuroimaging Initiative (ADNI) database (adni.loni.usc.edu). The ADNI was launched in 2003 as a public-private partnership, led by Principal Investigator Michael W. Weiner, MD. The primary goal of ADNI has been to test whether serial magnetic resonance imaging (MRI), positron emission tomography (PET), other biological markers, and clinical and neuropsychological assessment can be combined to measure the progression of mild cognitive impairment (MCI) and early Alzheimer's disease (AD). For up-to-date information, see http://www.adni-info.org.

Preprocessed [18F]Florbetapir PET scans (i.e., amyloid imaging data) were downloaded from adni.loni.usc.edu, then aligned to the corresponding MRI scans and normalized to the Montreal Neurological Institute (MNI) space as $2 \times 2 \times 2 \mathrm{~mm}$ voxels. ROI level amyloid measurements were further extracted based on the MarsBaR AAL atlas. Genotype data of both ADNI-1 and ADNI-GO/2 phases were also downloaded, and then quality controlled, imputed, and combined as described in [10]. A total of 980 nonHispanic Caucasian participants with both complete amyloid measurements and genome-wide data were studied. Associations between 105 (out of a total 116) baseline amyloid measures and 5,574,300 SNPs were examined by performing SNP-based GWAS using PLINK [17] with sex, age, and education as covariates. To facilitate the subsequent enrichment analysis, a gene-based $p$ value was determined as the smallest $p$ value of all SNPs located in $\pm 20 \mathrm{~K}$ bp of the gene [14].

\subsection{Constructing GS-BC modules using AHBA}

There are many types of prior knowledge that can be used to define meaningful GS and $\mathrm{BC}$ entities. In the genomic domain, the prior knowledge could be based on Gene Ontology or functional annotation databases; in the imaging domain, the prior knowledge could be neuroanatomic ontology or brain databases. In this work, to demonstrate the proposed IGEA framework, we use gene expression data from the Allen Human Brain Atlas (AHBA, Allen Institute for Brain Science, Seattle, WA, USA; available from http://www.brain-map.org/) to extract GS and BC modules such that genes within a GS share similar expression profiles and so do ROIs within a BC. We hypothesize that, given these similar co-expression patterns across genes and ROIs, each GS-BC pair forms an interesting high-level imaging genetic entity that may be related to certain biological function and can serve as a valuable candidate for two-dimensional IGEA. 
(A) SNP-level GWAS

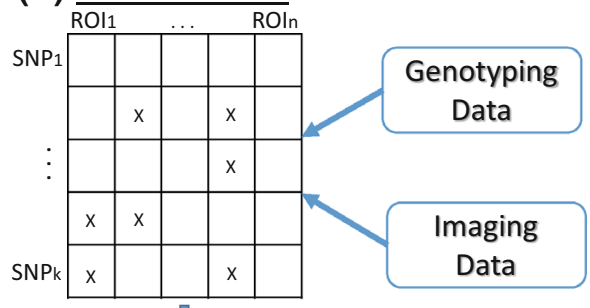

Mapping

(B) Gene-level GWAS

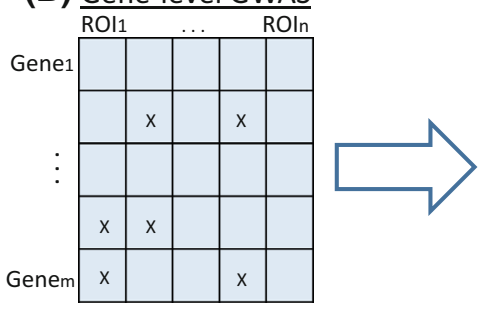

(C)

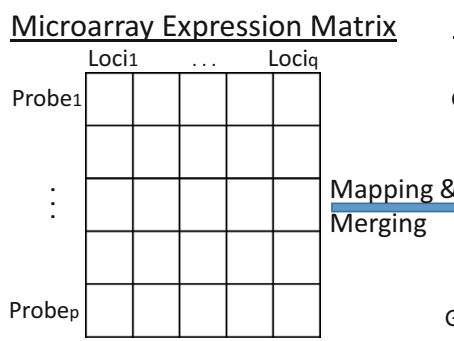

Gene-ROI Expression Matrix

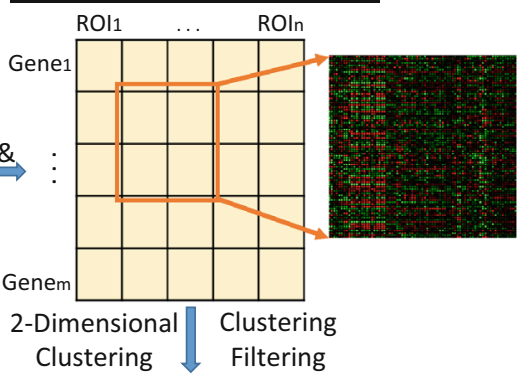

(D) Gene Set-Brain Circuit Modules

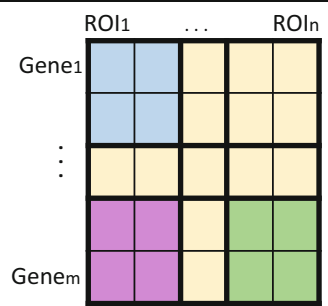

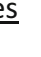

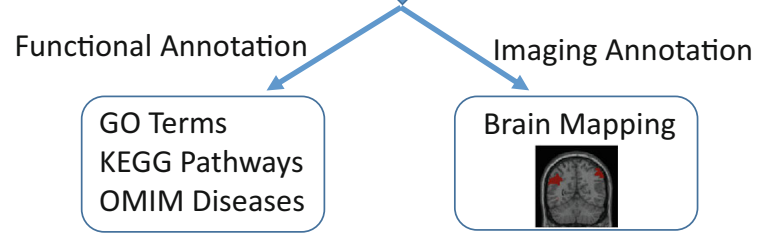

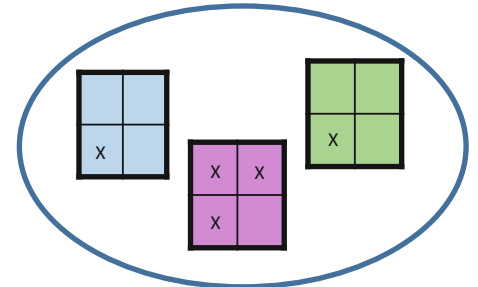

(F) Annotation

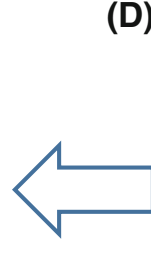

Genem 
Let $\mathbf{X}$ be a GS-BC module with $n$ genes and $m$ ROIs, where $\mathbf{x}^{i}$ is the expression level of gene $i$ across all the $m$ ROIs in $\mathbf{X}$, and $\mathbf{x}_{j}$ is the expression profile of ROI $j$ across all the $n$ genes in $\mathbf{X}$. For each pair of genes in $\mathbf{X}$, i.e., $\left(\left(\mathbf{x}^{i_{1}}\right)^{T},\left(\mathbf{x}^{i_{2}}\right)^{T}\right)$, we calculate its correlation coefficient. For each pair of ROIs in $\mathbf{X}$, i.e., $\left(\mathbf{x}_{j_{1}}, \mathbf{x}_{j_{2}}\right)$, we also calculate its correlation coefficient. After that, we transform each of these correlation coefficients, say $c$, to Fisher's $z$-statistic $z(c)$ using the following Eq. (3):

$z(c)=\frac{1}{2} \log \left(\frac{1+c}{1-c}\right)$.

We then define $\bar{z}_{\text {gene }}(\mathbf{X})$, the gene-based average Fisher's $z$-statistics of correlation coefficient of $\mathbf{X}$, as follows:

$\bar{z}_{\text {gene }}(\mathbf{X})=\frac{2}{n(n-1)} \sum_{0<i_{1}<i_{2} \leq n} z\left(\operatorname{corr}\left(\left(\mathbf{x}^{i_{1}}\right)^{T}\left(\mathbf{x}^{i_{2}}\right)^{T}\right)\right)$.

Similarly, we define $\bar{z}_{\text {roi }}(\mathbf{X})$, the ROI-based average Fisher's $z$-statistics of correlation coefficient of $\mathbf{X}$, as follows:

$\bar{z}_{\text {roi }}(\mathbf{X})=\frac{2}{m(m-1)} \sum_{0<j_{1}<j_{2} \leq m} z\left(\operatorname{corr}\left(\mathbf{x}_{j_{1}}, \mathbf{x}_{j_{2}}\right)\right)$.

Based on these average gene-based and ROI-based zstatistics, respectively, we select the top $20 \%$ of all the GS-BC modules and include those in our subsequent analyses, to ensure our studied modules have comparatively high co-expression profiles. Thus, in this work, we focus on the analysis of the following three types of GSBC modules with top $z$-statistics:

1. Gene-based These are the modules with relatively high co-expression profiles between genes, i.e., $\bar{z}_{\text {gene }}(\mathbf{X})$ is ranked in the top $20 \%$ of all the $\bar{z}_{\text {gene }}$ scores.

2. ROI-based These are the modules with relatively high co-expression profiles between ROIs, i.e., $\bar{z}_{\text {roi }}(\mathbf{X})$ is ranked in the top $20 \%$ of all the $\bar{z}_{\text {roi }}$ scores.

3. Gene and ROI-based Both (1) and (2) hold.

\subsection{Imaging Genetic Enrichment Analysis (IGEA)}

Pathway enrichment analysis has been extensively employed to genomic domain to analyze the genetic findings associated with a specific imaging QT. In this study, our goal is to identify high-level associations between gene sets and brain circuits, which typically include multiple genes and multiple QTs.

In this study, we propose the threshold-based IGEA by extending the existing threshold-based enrichment analysis. SNP-level findings have been mapped to gene level findings in Sect. 2.1. The GWAS findings are a list $L$ of $N=N_{G} \times N_{B}$ gene-QT associations, where we have a set
$G_{d}$ of $N_{G}=\left|G_{d}\right|$ genes and a set $B_{d}$ of $N_{B}=\left|B_{d}\right|$ QTs in our analysis. From Sect. 2.2, GS-BC modules have been constructed, where either relevant genes share similar expression profiles across relevant ROIs, or relevant ROIs share similar expression profiles across relevant genes, or both. Given an interesting GS-BC module with gene set $G_{k}$ and QT set $B_{k}$, IGEA aims to determine whether the target GS-BC module $T=\left\{(g, b) \mid g \in G_{d} \cap G_{k}, b \in B_{d} \cap B_{k}\right\}$ is enriched in $L$.

Now we describe our threshold-based IGEA method. We have $N$ gene-QT pairs from GWAS. Out of these, $n=|A|$ pairs (the set $A$ ) are significant ones with GWAS $p$ value passed a certain threshold. We also have $m=|P|$ (the set $P$ ) gene-QT pairs from a given GS-BC module, and $k$ significant pairs are from $P$. Using Fisher's exact test for independence, the enrichment $p$ value for the given GS-BC module is calculated as follows:

$p$-value $=\operatorname{Pr}(|A \cap P| \geq k)=\sum_{i \geq k} \frac{\left(\begin{array}{c}m \\ i\end{array}\right) \times\left(\begin{array}{c}N-m \\ n-i\end{array}\right)}{\left(\begin{array}{l}N \\ n\end{array}\right)}$.

Here, we use $\operatorname{Pr}(\cdot)$ to denote the probability function.

\subsection{Evaluation of the identified GS-BC modules}

For evaluation purpose, we tested the statistical significance of the IGEA results. We hypothesize that the geneQT associations from BWGWAS of the original data should be overrepresented in certain GS-BC modules, and the BWGWAS results on permuted data should not be enriched in a similar number of GS-BC modules. We performed the IGEA analysis on $n=50$ permuted BWGWAS results, and estimated the $p$ value for the number of significant GS-BC modules discovered from the original data using a $t$-distribution with $n-1$ degrees of freedom.

Given a BWGWAS result $R$, let $\operatorname{Prop}(R)$ be the proportion of modules which are significantly enriched by $R$. Let $R_{\text {orig }}$ be the original BWGWAS result, and $R_{\text {perm(i) }}$ be the $i$ th permuted BWGWAS result. Let $S=$ $\left\{\operatorname{Prop}\left(R_{\text {perm(i) }}\right) \mid 1 \leq i \leq n\right\}$ be the set of these proportion values for all the permuted results. Then the $p$ value is estimated using Eq. (7).

$p$-value $=\operatorname{Pr}\left(T_{n-1} \geq \frac{\operatorname{Prop}\left(R_{\text {orig }}\right)-\mu_{\text {perm }}}{\sqrt{1+1 / n} \times \sigma_{\text {perm }}}\right)$.

where $T_{n-1}$ is the $t$-distribution with $n-1$ degrees of freedom, $\mu_{\text {perm }}$ is the sample mean of $S$, and $\sigma_{\text {perm }}$ is the sample standard deviation of $S$.

To determine the functional relevance of the enriched GS-BC modules, we also tested whether genes from each module are overrepresented for specific neurobiological 


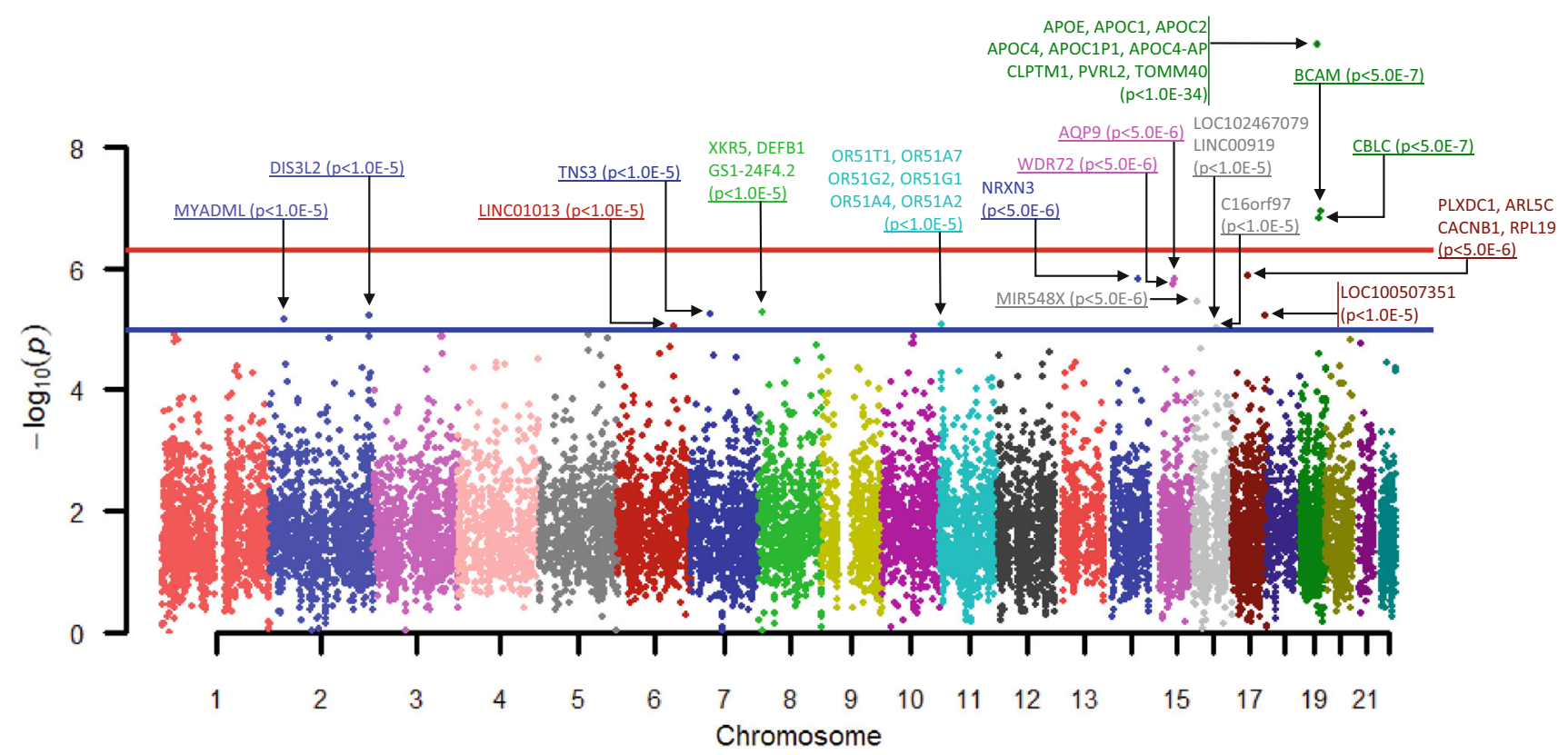

Fig. 2 Manhattan plot of imaging quantitative genome-wide association for Alzheimer's Disease individuals based on Precuneus (right) measurement from amyloid imaging data. The $x$ axis represents the

functions, signaling pathways, or complex neurodegenerative diseases. We performed pathway enrichment tests using gene ontology (GO) terms, KEGG pathways and OMIM (Online Mend-elian Inheritance in Man) database.

\section{Results}

\subsection{Significant GS-BC modules}

By performing hierarchical clustering on both genetic and imaging domains, 171 out of 216 genetic clusters (only those with size ranging from 10 to 200) and 9 imaging clusters (with size ranging from 4 to 23 , no clusters are excluded) were identified. 1539 GS-BC modules were generated by combining each pair of genetic and ROI clusters. Two sets of 308 (20\% of 1539) modules were selected according to gene-based and ROI-based z-statistics, respectively. Among them, 90 modules were among top $20 \%$ in both gene-based and ROI-based ranking results. We used a moderate size threshold for the selection, to avoid the exclusion of potentially interesting candidates.

For the BWGWAS results, we obtained $16,076 \times$ $105=1,687,980$ gene-QT associations after mapping SNP-based $p$ values to genes. Out of these, 1402 gene-QT associations passed the BWGWAS $p$ value of $1.0 \mathrm{e}-5$. Figure 2 shows the gene-based GWAS result of an chromosomes and the $y$ axis represents $-\log _{10}(P)$, where $P$ is the gene-based significance

example QT (i.e., the average amyloid deposition in the right precuneus). Precuneus amyloid concentration has been demonstrated to be associated with disordered activity in Alzheimer's Disease [8].

Three sets of constructed GS-BC modules $(308,308$, and 90 with top z-statistics using gene-based, ROI-based, and gene\&ROI-based strategies, respectively, see Sect. 2.2) were tested separately for whether they could be enriched by BWGWAS results using IGEA. Across three sets, totally 25 modules turned out to be significant after Bonferroni correction (see Table 1), of which 15, 17, and 9 are from gene-based, ROI-based, and both gene\&ROIbased categories, respectively. We also tested the significance of the number of identified GS-BC modules. Compared to the permuted BWGWAS results, the analysis on the original data yielded a significantly larger number of enriched GS-BC modules with estimated $p$ values of 7.6e-25, 1.2e-9, and 1.8e-25, corresponding to gene-based, ROI-based, and gene\&ROI-based strategies, respectively, indicating that imaging genetic associations existed in these enriched GS-BC modules.

Across all 25 identified modules, there are 9 and 8 unique GS and $\mathrm{BC}$ entities, respectively. Figure 3 shows the 8 unique identified $\mathrm{BCs}$ with corresponding ROI names, and Fig. 4 maps four of those onto the brain. For example, $\mathrm{BC} 03$ and $\mathrm{BC} 04$ include structures that are major spots for amyloid accumulation in $\mathrm{AD}$ (e.g., cingulum, precuneus). BC05 involves structures responsible for motivated behaviors (e.g., caudate, pallidum, putamen) and 
Table 1 Twenty-five significantly enriched GS-BC modules from IGEA

\begin{tabular}{|c|c|c|c|c|c|c|c|c|}
\hline $\begin{array}{l}\text { Module } \\
\text { ID }\end{array}$ & $\begin{array}{l}\text { Top } 20 \% \text { Co- } \\
\text { expressed }^{\text {a }}\end{array}$ & BC ID & $\begin{array}{l}\text { \# of } \\
\text { ROIs }\end{array}$ & GS ID & $\begin{array}{l}\# \text { of } \\
\text { genes }\end{array}$ & $\begin{array}{l}\text { Corrected } P \text { value } \\
\text { (gene-based) }\end{array}$ & $\begin{array}{l}\text { Corrected } P \text { value } \\
\text { (ROI-based) }\end{array}$ & $\begin{array}{l}\text { Corrected } P \text { value } \\
\text { (Gene\&ROI-based) }\end{array}$ \\
\hline 01 & $\mathrm{R}^{\mathrm{c}}$ & ВC07 & 8 & GS01 & 81 & - & $2.61 \mathrm{E}-06$ & - \\
\hline 02 & $\mathrm{G}, \mathrm{R}, \mathrm{G} \& \mathrm{R}^{\mathrm{d}}$ & ВC02 & 4 & GS02 & 168 & $9.06 \mathrm{E}-06$ & $9.06 \mathrm{E}-06$ & $9.06 \mathrm{E}-06$ \\
\hline 03 & $\mathrm{G}^{\mathrm{b}}$ & $\mathrm{BC} 03$ & 11 & GS02 & 168 & $2.54 \mathrm{E}-11$ & - & - \\
\hline 04 & $\mathrm{G}, \mathrm{R}, \mathrm{G} \& \mathrm{R}$ & $\mathrm{BC} 04$ & 5 & GS02 & 168 & $1.44 \mathrm{E}-06$ & $1.44 \mathrm{E}-06$ & $1.44 \mathrm{E}-06$ \\
\hline 05 & G & BC05 & 14 & GS02 & 168 & $6.42 \mathrm{E}-06$ & - & - \\
\hline 06 & $\mathrm{R}$ & ВC06 & 13 & GS02 & 168 & - & $5.91 \mathrm{E}-07$ & - \\
\hline 07 & $\mathrm{R}$ & BC08 & 23 & GS02 & 168 & - & $5.65 \mathrm{E}-22$ & - \\
\hline 08 & $\mathrm{G}, \mathrm{R}, \mathrm{G} \& \mathrm{R}$ & $\mathrm{BC} 01$ & 4 & GS03 & 55 & $1.38 \mathrm{E}-06$ & $1.38 \mathrm{E}-06$ & $1.38 \mathrm{E}-06$ \\
\hline 09 & G & $\mathrm{BC} 02$ & 4 & GS03 & 55 & $4.39 \mathrm{E}-13$ & - & - \\
\hline 10 & $\mathrm{R}$ & $\mathrm{BC} 04$ & 5 & GS03 & 55 & - & $1.41 \mathrm{E}-15$ & - \\
\hline 11 & G & BC05 & 14 & GS03 & 55 & $1.01 \mathrm{E}-14$ & - & - \\
\hline 12 & $\mathrm{R}$ & ВC06 & 13 & GS03 & 55 & - & $1.72 \mathrm{E}-08$ & - \\
\hline 13 & $\mathrm{R}$ & $\mathrm{BC} 07$ & 8 & GS03 & 55 & - & $2.40 \mathrm{E}-21$ & - \\
\hline 14 & $\mathrm{R}$ & $\mathrm{BC} 07$ & 8 & GS04 & 66 & - & $4.00 \mathrm{E}-07$ & - \\
\hline 15 & $\mathrm{G}, \mathrm{R}, \mathrm{G} \& \mathrm{R}$ & BC01 & 4 & GS05 & 19 & $3.83 \mathrm{E}-05$ & $3.83 \mathrm{E}-05$ & $3.83 \mathrm{E}-05$ \\
\hline 16 & $\mathrm{G}, \mathrm{R}, \mathrm{G} \& \mathrm{R}$ & $\mathrm{BC} 02$ & 4 & GS05 & 19 & $6.88 \mathrm{E}-09$ & $6.88 \mathrm{E}-09$ & $6.88 \mathrm{E}-09$ \\
\hline 17 & $\mathrm{G}, \mathrm{R}, \mathrm{G} \& \mathrm{R}$ & $\mathrm{BC} 04$ & 5 & GS05 & 19 & $2.64 \mathrm{E}-10$ & $2.64 \mathrm{E}-10$ & $2.64 \mathrm{E}-10$ \\
\hline 18 & $\mathrm{R}$ & BC06 & 13 & GS05 & 19 & - & $2.26 \mathrm{E}-11$ & - \\
\hline 19 & $\mathrm{G}, \mathrm{R}, \mathrm{G} \& \mathrm{R}$ & $\mathrm{BC} 07$ & 8 & GS05 & 19 & $1.54 \mathrm{E}-14$ & $1.54 \mathrm{E}-14$ & $1.54 \mathrm{E}-14$ \\
\hline 20 & $\mathrm{G}, \mathrm{R}, \mathrm{G} \& \mathrm{R}$ & $\mathrm{BC} 02$ & 4 & GS06 & 28 & $4.87 \mathrm{E}-08$ & $4.87 \mathrm{E}-08$ & $4.87 \mathrm{E}-08$ \\
\hline 21 & $\mathrm{G}$ & $\mathrm{BC} 02$ & 4 & GS07 & 24 & $7.69 \mathrm{E}-05$ & - & - \\
\hline 22 & G\&R & $\mathrm{BC} 01$ & 4 & GS08 & 33 & - & - & $1.97 \mathrm{E}-04$ \\
\hline 23 & $\mathrm{G}$ & $\mathrm{BC} 02$ & 4 & GS08 & 33 & $1.11 \mathrm{E}-07$ & - & - \\
\hline 24 & $\mathrm{R}$ & $\mathrm{BC} 04$ & 5 & GS08 & 33 & - & $7.39 \mathrm{E}-09$ & - \\
\hline 25 & $\mathrm{G}$ & $\mathrm{BC} 02$ & 4 & GS09 & 111 & $4.07 \mathrm{E}-05$ & - & - \\
\hline
\end{tabular}

See also Sect. 3.2 and Fig. 3 for details about relevant GSs and BCs, respectively

${ }^{\text {a }}$ To indicate whether the top $20 \%$ modules are selected based on the gene-based, ROI-based, or gene\&ROI-based strategy

b G: Gene-based

c R: ROI-based

d G\&R: Gene\&ROI-based

sensory information processing (e.g., thalamus). BC08 involves various frontal regions responsible for executive functions. Details of all 25 modules are listed in Table 1. We can find that some modules share common gene sets with different brain circuits, and some share the same brain circuits with different gene sets. This illustrates the complex associations among multiple genes and multiple brain ROIs.

\subsection{Pathway analysis of identified GS-BC modules}

To explore and analyze functional relevance of our identified GS-BC modules, we performed pathway enrichment analysis from three aspects including GO terms, functional pathways and diseases using Gene Ontology (GO), KEGG pathways, and OMIM diseases databases, respectively.
Figure 5 shows the KEGG pathway enrichment results which were mapped to 15 categories. From the results, most identified GSs had a number of significant functional enrichments. Several of them were directly related to the neurodegenerative disease and its development, e.g., Alzheimer's Disease enriched in GS05 and Parkinson's Disease enriched in GS01. Another major part of them were also related to the neurodegenerative diseases and their development. For instance, caffeine as the most widely used psychoactive substance, its metabolism (from GS09 located in Module 25) can affect brain metabolism and has potential benefits on Parkinson's Disease treatment [16]. There are also several enriched pathways related to oxidative stress, which is a critical factor for a range of neurodegenerative disorders. For example, glycolysis and gluconeogenesis (from GS02 located in Modules 02-07) 


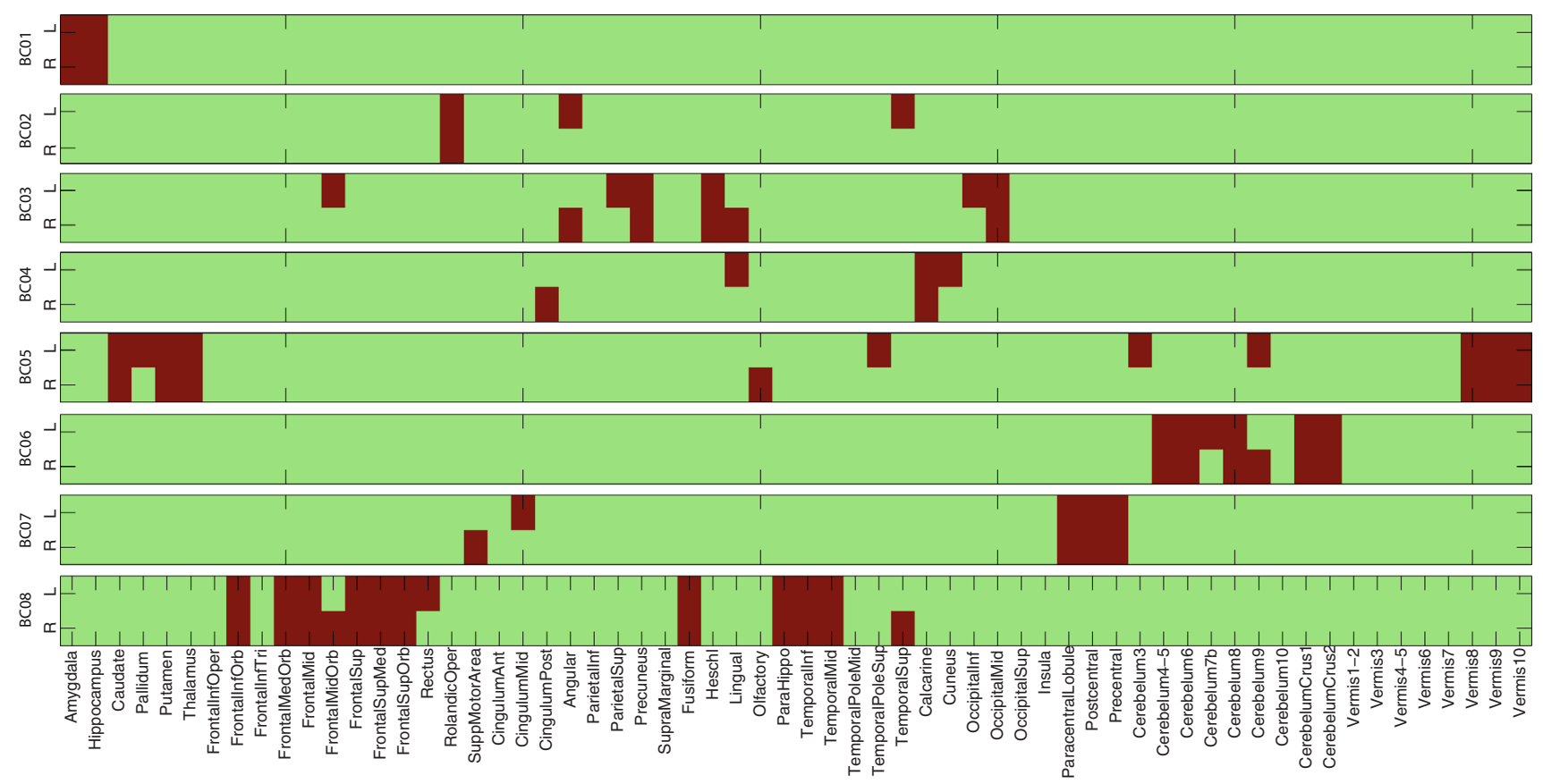

Fig. 3 Eight unique brain circuits (BCs) identified from IGEA. ROIs belonging to each BC are colored in red
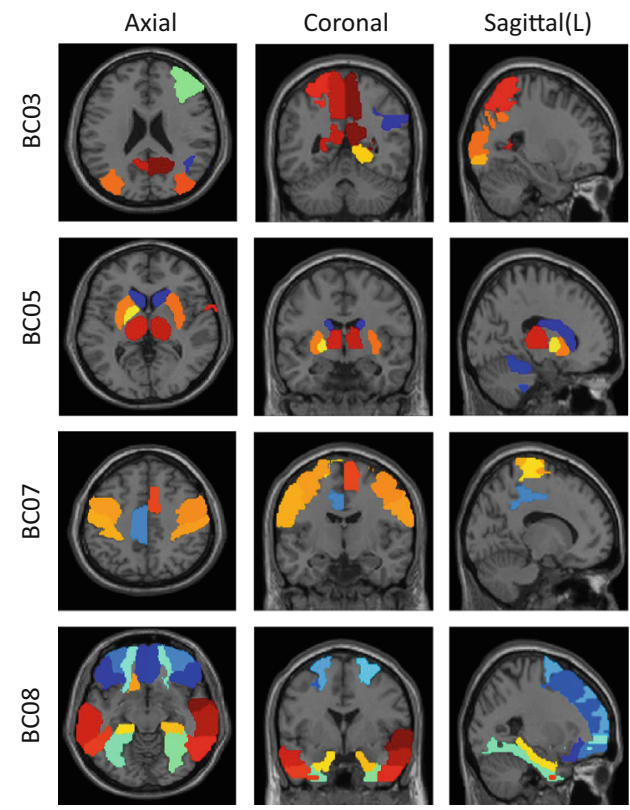

Fig. 4 Brain maps of four brain circuits (BCs) identified from IGEA

are associated with hypoxia, ischemia, and AD [2]. Gap junctions (from GS03 located in Modules 08-13) can couple various kinds of cells in the central nervous system (CNS) which play an important role in maintaining normal function. Signaling transduction, like calcium signaling pathway (from GS03 located in Modules 08-13) playing key role in short- and long-term synaptic plasticity has shown abnormality in many neurodegenerative disorders including Alzheimer's Disease, Parkinson's disease, amyotrophic lateral sclerosis (ALS), Huntington's disease, spinocerebellar ataxias (SCA), and so on [1].

Table 2 shows the OMIM disease enrichment results. Several neurodegeneration-related and age-related diseases and complex disorders were enriched in various gene sets, such as Alzheimer's Diease from GS03 and GS05, Encephalopathy from GS01 and GS02, and Anomalies from GS05. Besides neurodegeneration diseases and disorders, several cancer-related entities are detected including breast cancer from GS02 and leukemia from GS03. These findings provided potential evidence for the studies that focused on investigating the relationship between cancer and neurodegeneration, with abnormal cell growth and cell loss in common.

Gene Ontology (GO) enrichment indicates the relationship between identified gene sets and GO terms from three categories including biological process (BP), cellular component (CC), and molecular function (MF) (http://gen eontology.org/). For the GO enrichment of all 9 gene sets, 163 various GO terms were significantly enriched. Top enriched terms were selected and grouped to 7 categories including behavior, cell communication, mitochondrion, metabolic process, neurological system process, response to stimulus, and signal transduction, as shown in Table 3 . A large number of these terms have direct or indirect relationships with neurodegenerative diseases or phenotypes. 


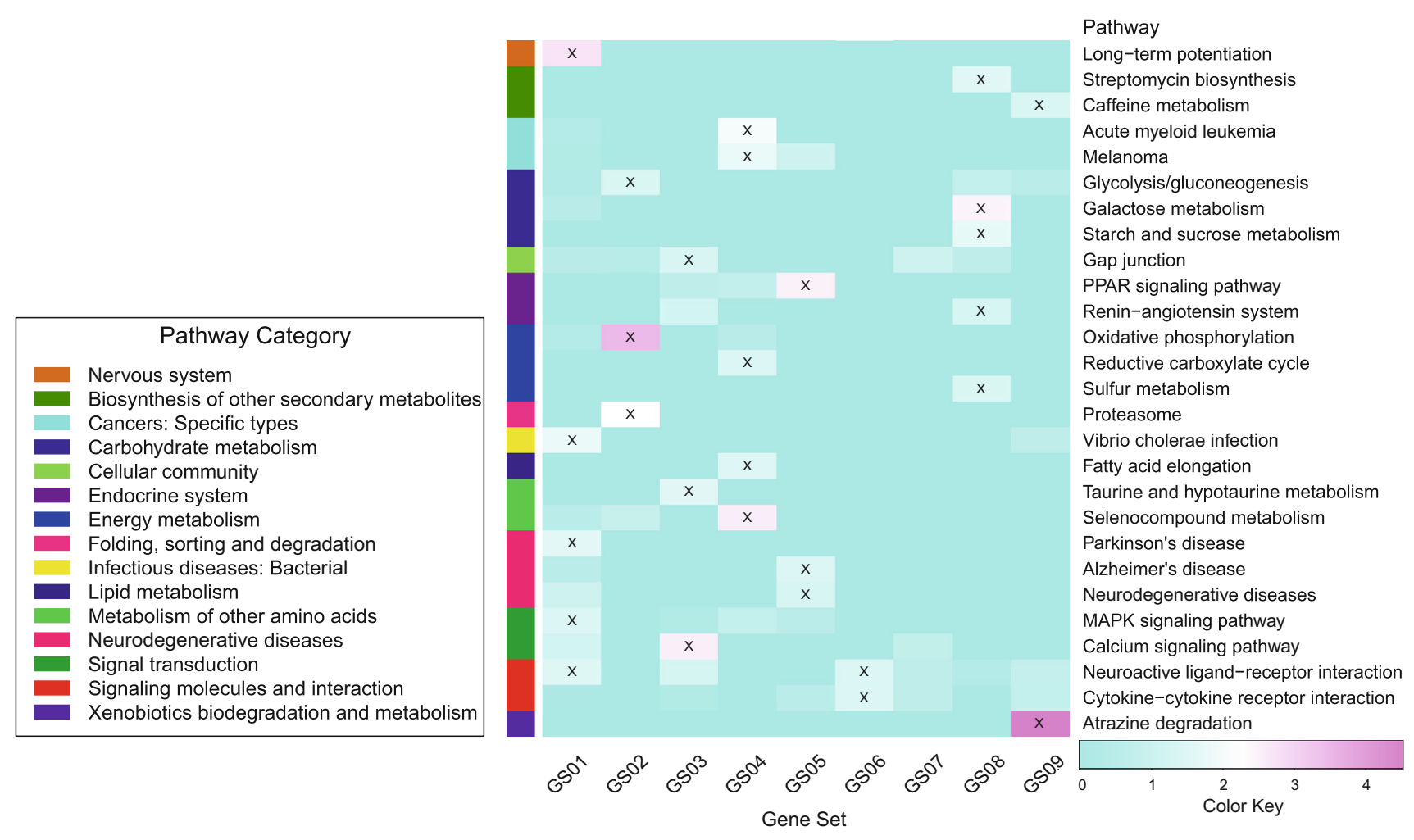

Fig. 5 Results of KEGG pathway enrichment for identified GSs. The $x$ axis represents unique GS ID, and $y$ axis represents $-\log p$ value of enrichment significance of KEGG pathways. Marked cell represents significant enrichment ( $p$ value $<0.05)$

Table 2 Top enriched OMIM diseases of identified GSs

\begin{tabular}{|c|c|c|c|}
\hline GS ID & \# of gene & OMIM Disease & $P$ value \\
\hline \multirow[t]{2}{*}{ GS01 } & 81 & Encephalopathy & $4.2 \mathrm{E}-2^{*}$ \\
\hline & & Dementia & $3.6 \mathrm{E}-2 *$ \\
\hline \multirow[t]{2}{*}{ GS02 } & 168 & Encephalopathy & $5.0 \mathrm{E}-2$ \\
\hline & & Breast cancer & $9.5 \mathrm{E}-2$ \\
\hline \multirow[t]{2}{*}{ GS03 } & 55 & Leukemia & $2.7 \mathrm{E}-2 *$ \\
\hline & & Alzheimer's disease & $8.9 \mathrm{E}-2$ \\
\hline GS04 & 66 & Hypertension & $5.0 \mathrm{E}-2$ \\
\hline \multirow[t]{2}{*}{ GS05 } & 19 & Anomalies & $2.4 \mathrm{E}-2^{*}$ \\
\hline & & Alzheimer's disease & $4.5 \mathrm{E}-2 *$ \\
\hline GS06 & 28 & Ectodermal dysplasia & $2.0 \mathrm{E}-2 *$ \\
\hline \multirow[t]{2}{*}{ GS07 } & 24 & Hypertension & $3.4 \mathrm{E}-2 *$ \\
\hline & & Spinocerebellar ataxia & $4.3 \mathrm{E}-2^{*}$ \\
\hline GS08 & 33 & Glycogen storage disease & $1.6 \mathrm{E}-2 *$ \\
\hline GS09 & 111 & Immunodeficiency & $1.4 \mathrm{E}-2 *$ \\
\hline
\end{tabular}

* Significantly enriched

\section{Discussion}

We have presented a two-dimensional imaging genetic enrichment analysis (IGEA) framework to explore the high-level imaging genetic associations by integrating whole brain genomic, transcriptomic, and neuroanatomic data. Traditional pathway enrichment analysis focused on investigating genetic findings of a single phenotype one at a time, and relationships among imaging QTs could be ignored. Such approach could be inadequate to provide insights into the mechanisms of complex diseases that involve multiple genes and multiple QTs. In this paper, we have proposed a novel enrichment analysis paradigm IGEA to detect high-level associations between gene sets and brain circuits. By jointly considering the complex relationships between interlinked genetic markers and correlated brain imaging phenotypes, IGEA provides additional power for extracting biological insights on neurogenomic associations at a systems biology level. For example, let us take a look at GS03-BC05, an identified module significantly enriched by our GWAS findings. Several ROIs (e.g., caudate, pallidum, and putamen) from $\mathrm{BC} 05$ have been indicated responsible for motivated behaviors [3]. Meanwhile both KEGG and GO functional enrichment results of GS03 show high relevance to behavior and normal function maintaining (see Fig. 5; Table 3). These observations suggest that this high-level imaging genetic pattern could be relevant to the behavior mechanism. It warrants further investigation to perform analyses targeted at these genes and ROIs in independent cohorts to better understand the underlying mechanism from the imaging genetic perspective. 
Table 3 Top enriched GO terms of GSs from identified GS-BC modules

\begin{tabular}{|c|c|c|c|c|}
\hline Group & GS ID & \# of genes & GO Category & Corrected $p$ value \\
\hline \multirow[t]{2}{*}{ Behavior } & GS03 & 55 & Behavior & $2.2 \mathrm{E}-2$ \\
\hline & & & Learning or memory & $4.4 \mathrm{E}-2$ \\
\hline \multirow[t]{3}{*}{ Cell communication } & GS01 & 81 & Regulation of synaptic transmission & $2.7 \mathrm{E}-6$ \\
\hline & & & Neuron-neuron Synaptic transmission & $2.9 \mathrm{E}-3$ \\
\hline & GS03 & 55 & Synaptic transmission & $1.7 \mathrm{E}-4$ \\
\hline \multirow[t]{3}{*}{ Metabolic process } & GS05 & 19 & Fat-soluble vitamin metabolic process & $4.3 \mathrm{E}-2$ \\
\hline & & & Organic hydroxy compound biosynthetic process & $4.8 \mathrm{E}-2$ \\
\hline & GS06 & 28 & Regulation of translational termination & $2.8 \mathrm{E}-2$ \\
\hline \multirow[t]{2}{*}{ Mitochondrion } & GS02 & 168 & Mitochondrial membrane part & $2.5 \mathrm{E}-3$ \\
\hline & & & Mitochondrial respiratory chain complex I & $4.9 \mathrm{E}-3$ \\
\hline \multirow[t]{4}{*}{ Neurological system process } & GS03 & 55 & Associative learning & $1.1 \mathrm{E}-2$ \\
\hline & & & Learning & $4.5 \mathrm{E}-6$ \\
\hline & GS09 & 111 & Detection of chemical stimulus involved in sensory perception & $1.1 \mathrm{E}-4$ \\
\hline & & & Olfactory receptor activity & $1.9 \mathrm{E}-5$ \\
\hline \multirow[t]{5}{*}{ Response to stimulus } & GS03 & 55 & Response to amphetamine & $2.0 \mathrm{E}-3$ \\
\hline & & & Visual behavior & $4.5 \mathrm{E}-3$ \\
\hline & GS05 & 19 & Response to cholesterol & $3.6 \mathrm{E}-2$ \\
\hline & & & Response to sterol & $3.7 \mathrm{E}-2$ \\
\hline & GS09 & 111 & Detection of chemical stimulus & $1.6 \mathrm{E}-4$ \\
\hline \multirow[t]{5}{*}{ Signal transduction } & GS01 & 81 & Glutamate receptor signaling pathway & $7.3 \mathrm{E}-4$ \\
\hline & GS03 & 55 & Adenylate cyclase-activating dopamine receptor signaling pathway & $3.1 \mathrm{E}-3$ \\
\hline & & & Dopamine receptor signaling pathway & $1.4 \mathrm{E}-2$ \\
\hline & GS05 & 19 & Transmembrane receptor protein kinase activity & $4.4 \mathrm{E}-2$ \\
\hline & GS09 & 111 & Olfactory receptor activity & $1.9 \mathrm{E}-5$ \\
\hline
\end{tabular}

The real power of IGEA, however, can be affected by several aspects. First, the constructed GS-BC modules should reflect the real relationships among genes as well as brain ROIs. Thus, it is crucial to define meaningful gene sets and brain circuits. In our paper, GSs and BCs were separately extracted from AHBA brain wide expression data based on hierarchical clustering, which were then combined to provide GS-BC modules. This strategy was based on the idea that interlinked genetic markers (or brain ROIs) would conserve similar expression pattern, i.e., would be highly co-expressed. Second, the statistical measure of enrichment evaluation can be based on different strategies. We adopted hypergeometric test in our experiment to estimate the over-representation of our defined GS-BC modules to the list of gene-QT pair.

Based on these two considerations, our proposed paradigm can be further improved. From our GS-BC module construction, GSs (or BCs) are clustered together based on their co-expression pattern across all the ROIs in the whole brain (or across all the genes in the genome). Although statistical measures were calculated using Fisher's z-transformation to restrict our analyses on only highly coexpressed modules from our bi-clustering results, we could be missing other highly co-expressed GSs (or BCs) if they only had similar expression patterns on a small set of ROIs (or genes). In other words, our module construction strategy considered the global expression pattern but ignored the local ones. It is worth for further investigation to try other reasonable strategies by applying prior knowledge such as pre-defined genetic pathways/networks or brain circuits, or by using different co-clustering algorithms (e.g., [26]) to take into consideration of relevant local expression patterns.

Hypergeometric test requires a pre-defined threshold to determine the list of gene-QT pairs. Another limitation is that it considers only the count of significant gene-QT pairs, but ignores the strength of gene-QT associations. There are a number of rank-based enrichment analysis methods (e.g., GSEA [23]) that can be employed in our two-dimensional enrichment analysis to overcome these disadvantages. Another issue is that we used the smallest SNP-level $p$ value within the gene to represent the genebased $p$ value. Therefore, another possible future direction is to explore other set-based methods for calculating genebased $p$ values such as VEGAS [13], GATES [12], and so on. Besides, from mathematical perspective, associating 
GS-BC modules and gene-QT findings can be seen as a similarity discovery over two matrices. Thus, another future direction could be to study this problem using machine learning approaches similar to that proposed by Wang et al. [25].

Acknowledgments At Indiana University, this work was supported by NIH R01 LM011360, U01 AG024904, RC2 AG036535, R01 AG19771, P30 AG10133, UL1 TR001108, R01 AG 042437, R01 AG046171, R00 LM011384, and R03 AG050856; NSF IIS-1117335; DOD W81XWH-14-2-0151, W81XWH-13-1-0259, and W81XWH12-2-0012; NCAA 14132004; and CTSI SPARC Program. At University of Pennsylvania, the work was supported by NIH R01 LM011360, R01 LM009012, and R01 LM010098. Data collection and sharing for this project was funded by the Alzheimer's Disease Neuroimaging Initiative (ADNI) (National Institutes of Health Grant U01 AG024904) and DOD ADNI (Department of Defense award number W81XWH-12-2-0012). ADNI is funded by the National Institute on Aging, the National Institute of Biomedical Imaging and Bioengineering, and through generous contributions from the following: AbbVie, Alzheimer's Association; Alzheimer's Drug Discovery Foundation; Araclon Biotech; BioClinica, Inc.; Biogen; Bristol-Myers Squibb Company; CereSpir, Inc.; Eisai Inc.; Elan Pharmaceuticals, Inc.; Eli Lilly and Company; EUROIMMUN; F. Hoffmann-La Roche Ltd and its affiliated company Genentech, Inc.; Fujirebio; GE Healthcare; IXICO Ltd.; Janssen Alzheimer Immunotherapy Research \& Development, LLC.; Johnson \& Johnson Pharmaceutical Research \& Development LLC.; Lumosity; Lundbeck; Merck \& Co., Inc.; Meso Scale Diagnostics, LLC.; NeuroRx Research; Neurotrack Technologies; Novartis Pharmaceuticals Corporation; Pfizer Inc.; Piramal Imaging; Servier; Takeda Pharmaceutical Company; and Transition Therapeutics. The Canadian Institutes of Health Research is providing funds to support ADNI clinical sites in Canada. Private sector contributions are facilitated by the Foundation for the National Institutes of Health (http://www.fnih.org). The grantee organization is the Northern California Institute for Research and Education, and the study is coordinated by the Alzheimer's Disease Cooperative Study at the University of California, San Diego. ADNI data are disseminated by the Laboratory for Neuro Imaging at the University of Southern California.

\section{Compliance with ethical standards}

Conflict of interest The authors declare no conflict of interest.

Compliance with ethical standards Appropriate Institutional Review Boards approval occurred at each ADNI site and informed consent was obtained from each participant or authorized representative.

Open Access This article is distributed under the terms of the Creative Commons Attribution 4.0 International License (http://crea tivecommons.org/licenses/by/4.0/), which permits unrestricted use, distribution, and reproduction in any medium, provided you give appropriate credit to the original author(s) and the source, provide a link to the Creative Commons license, and indicate if changes were made.

\section{References}

1. Bezprozvanny I (2009) Calcium signaling and neurodegenerative diseases. Trends Mol Med 15(3):89-100
2. Butterfield D, Lange M (2009) Multifunctional roles of enolase in Alzheimer's disease brain: beyond altered glucose metabolism. J Neurochem 111(4):915-33

3. Delgado MR, Stenger VA, Fiez JA (2004) Motivation-dependent responses in the human caudate nucleus. Cereb Cortex 14(9): 1022-30

4. Draghici S, Khatri P et al (2003) Global functional profiling of gene expression. Genomics 81(2):98-104

5. Draghici S, Khatri P et al (2003) Onto-tools, the toolkit of the modern biologist: onto-express, onto-compare, onto-design and onto-translate. Nucleic Acids Res 31(13):3775-3781

6. Hirschhorn JN (2009) Genomewide association studies-illuminating biologic pathways. N Engl J Med 360(17):1699-1701

7. Hong MG, Alexeyenko A et al (2010) Genome-wide pathway analysis implicates intracellular transmembrane protein transport in Alzheimer disease. J Hum Genet 55(10):707-709

8. Ikonomovic MD, Klunk WE, Abrahamson EE, Wuu J, Mathis CA, Scheff SW, Mufson EJ, DeKosky ST (2011) Precuneus amyloid burden is associated with reduced cholinergic activity in Alzheimer disease. Neurology 77(1):39-47

9. Khatri P, Draghici S (2005) Ontological analysis of gene expression data: current tools, limitations, and open problems. Bioinformatics 21(18):3587-3595

10. Kim S, Swaminathan $S$ et al (2013) Influence of genetic variation on plasma protein levels in older adults using a multi-analyte panel. Plos One 8(7):e70269

11. Lambert JC, Grenier-Boley B et al (2010) Implication of the immune system in Alzheimer's disease: evidence from genomewide pathway analysis. J Alzheimers Dis 20(4):1107-1118

12. Li MX, Gui HS, Kwan JS, Sham PC (2011) Gates: a rapid and powerful gene-based association test using extended Simes procedure. Am J Hum Genet 88(3):283-293

13. Liu JZ, McRae AF, Nyholt DR, Medland SE, Wray NR, Brown KM, Investigators A, Hayward NK, Montgomery GW, Visscher PM, Martin NG, Macgregor S (2010) A versatile gene-based test for genome-wide association studies. Am J Hum Genet 87(1):139-145

14. Nam D, Kim J, Kim SY, Kim S (2010) Gsa-snp: a general approach for gene set analysis of polymorphisms. Nucleic Acids Res 38:W749-W754

15. O'Dushlaine C, Kenny E et al (2011) Molecular pathways involved in neuronal cell adhesion and membrane scaffolding contribute to schizophrenia and bipolar disorder susceptibility. Mol Psychiatry 16(3):286-292

16. Postuma RB, Lang AE, Munhoz RP, Charland K, Pelletier A, Moscovich M, Filla L, Zanatta D, Rios Romenets S, Altman R, Chuang R, Shah B (2012) Caffeine for treatment of parkinson disease: a randomized controlled trial. Neurology 79(7):651-658

17. Purcell S, Neale B et al (2007) Plink: a tool set for whole-genome association and population-based linkage analyses. Am J Hum Genet 81(3):559-575

18. Ramanan V, Shen L et al (2012) Pathway analysis of genomic data: concepts, methods, and prospects for future development. Trends Genet 28(7):323-332

19. Ramanan VK, Kim S et al (2012) Genome-wide pathway analysis of memory impairment in the Alzheimer's Disease Neuroimaging Initiative (ADNI) cohort implicates gene candidates, canonical pathways, and networks. Brain Imaging Behav 6(4):634-648

20. Saykin AJ, Shen L et al (2010) Alzheimer's Disease Neuroimaging Initiative biomarkers as quantitative phenotypes: genetics core aims, progress, and plans. Alzheimers Dement 6(3):265-273

21. Shen L, Thompson PM et al (2014) Genetic analysis of quantitative phenotypes in $\mathrm{AD}$ and $\mathrm{MCI}$ : imaging, cognition and biomarkers. Brain Imaging Behav 8(2):183-207

22. Sokal R, Michener C (1958) A statistical method for evaluating systematic relationships. Univ Kansas Sci Bull 38:1409-1438 
23. Subramanian A, Tamayo P et al (2005) Gene set enrichment analysis: a knowledge-based approach for interpreting genome-wide expression profiles. Proc Natl Acad Sci USA 102(43):15545-15550

24. Ulitsky I, Maron-Katz A et al (2010) Expander: from expression microarrays to networks and functions. Nature Protocols 5(2):303-322

25. Wang H, Huang H, Ding C (2016) Correlated protein function prediction via maximization of data-knowledge consistency. RECOMB'14

26. Wang H, Nie F, Huang H, Makedon F (2016) Fast nonnegative matrix tri-factorization for large-scale data co-clustering. In: Proceedings of 22rd international joint conference on artificial intelligence (IJCAI'11)

27. Yan J, Du L et al (2014) Transcriptome-guided amyloid imaging genetic analysis via a novel structured sparse learning algorithm. Bioinformatics 30(17):i564-71

28. Zeng H, Shen EH et al (2012) Large-scale cellular-resolution gene profiling in human neocortex reveals species-specific molecular signatures. Cell 149(2):483-96

Xiaohui Yao holds a B.S. degree in Computer Science and Technology from Qing Dao University, and an M.S. degree in Computer Software and Theory from University of Science and Technology of China. She is currently pursuing her Ph.D. degree in Bioinformatics at Indiana University-Purdue University Indianapolis, and is a Research Assistant in Center for Neuroimaging at Indiana University School of Medicine. Her research interests include imaging genetics, multidimensional data mining, and information visualization.

Jingwen Yan holds a B.S. degree in Information Engineering from Nanjing University of Aeronautics and Astronautics, an M.S. degree in Pattern Recognition and Intelligence System from Huazhong University of Science and Technology, and a Ph.D. degree in Bioinformatics from Indiana University. She is an Assistant Research Scientist at Indiana University Network Science Institute and an Adjunct Assistant Research Professor at Indiana University School of Medicine. She will become an Assistant Professor of Bio-Health Informatics at Indiana University-Purdue University Indianapolis in August 2016. Her research interests include machine learning and its application to imaging genetics, next generation sequencing data analysis, and network approaches in integrative multi-omics analysis.

Sungeun Kim holds a B.S. degree in Electronic Engineering from Korea University, an M.S. degree in Radio Sciences and Engineering from Korea University, and a Ph.D. degree in Electrical and Computer Engineering from Purdue University. He is an Assistant Research Professor of Radiology and Imaging Sciences at Indiana University School of Medicine. His research interest is to develop computational methods for analyzing medical imaging, biomarker, and genetics data to expedite early detection and diagnosis of neurodegenerative diseases and to better understand the development of those diseases. He is also interested in studies on the neurological basis of language processing and visual perception.

Kwangsik Nho holds a B.S. degree in Physics from Korea University, an M.S. degree in Physics from Korea University, and a Ph.D. degree in Computational Physics from Florida State University. He is an Assistant Professor of Radiology and Imaging Sciences at Indiana University School of Medicine. His research interests include brain imaging genomics, data (text) mining, computational physics, and integration of bioinformatics, imaging informatics, and medical informatics.
Shannon L. Risacher holds a B.S. degree in Psychology from Indiana University-Purdue University Indianapolis, and a Ph.D. degree in Medical Neuroscience from Indiana University School of Medicine. She is an Assistant Professor of Radiology and Imaging Sciences at Indiana University School of Medicine. Her main research interests involve evaluating imaging and non-imaging biomarkers of Alzheimer's disease (AD) for utility in early detection and diagnosis. In particular, she is interested in evaluating which biomarkers are most sensitive in the earliest stages of disease, both for detecting pathophysiological changes and for predicting future clinical outcomes. She is primarily focused on structural, functional, and molecular imaging biomarkers of $\mathrm{AD}$, but has an additional interest in novel biomarkers such as sensory and perceptual tests.

Mark Inlow holds a B.A. degree in Mathematics from DePauw University, an M.S. degree in Statistics from San Diego State University, and a Ph.D. degree in Statistics from Texas A\&M University. $\mathrm{He}$ is a Lead Analyst at the American Institutes for Research and an Adjunct Associate Professor of Radiology and Imaging Sciences at Indiana University School of Medicine. His research interests include statistical methods for neuroimaging genomics, large-scale inference, multiple comparisons, and regression models with errors in variables.

Jason H. Moore holds a B.S. degree in Biological Sciences from Florida State University, and an M.S. degree in Human Genetics, an M.A. degree in Applied Statistics, and a Ph.D. degree in Human Genetics from University of Michigan Ann Arbor. He is the Edward Rose Professor of Informatics in Department of Biostatistics and Epidemiology at Perelman School of Medicine in University of Pennsylvania, and the Director of Penn Institute for Biomedical Informatics. He has a strong track record developing, evaluating, and applying computational algorithms and software for identifying genetic, genomic, and proteomic biomarkers of human health and disease. His central focus has been on developing computational methods that embrace, rather than ignore, the complexity of human biology.

Andrew J. Saykin holds a B.A. degree in Psychology from University of Massachusetts Amherst, and an M.S. degree in Clinical Psychology and a Psy.D. degree in Clinical Neuropsychology from Hahnemann Medical College. He is the Raymond C. Beeler Professor of Radiology and Professor of Medical and Molecular Genetics at Indiana University School of Medicine. His expertise is in the areas of multimodal neuroimaging research, human genetics, and neuropsychology/ cognitive neuroscience. He has a longstanding interest in the structural, functional, and molecular substrates of cognitive deficits in Alzheimer's disease, cancer, brain injury, schizophrenia, and other neurological and neuropsychiatric disorders. The major thrust of his current research program is on integrating advanced brain imaging and genomic data to enhance the understanding of disorders affecting memory.

Li Shen holds a B.S. degree from Xi' an Jiao Tong University, an M.S. degree from Shanghai Jiao Tong University, and a Ph.D. degree from Dartmouth College, all in Computer Science. He is an Associate Professor of Radiology and Imaging Sciences at Indiana University School of Medicine. His research interests include medical image computing, bioinformatics, data mining, network science, systems biology, brain imaging genomics, and brain connectomics. 